\title{
Enzymatically Modified Low-Density Lipoprotein Is Recognized by C1q and Activates the Classical Complement Pathway
}

\author{
Gérard J. Arlaud, ${ }^{1}$ Adrienn Biro, ${ }^{1}$ and Wai Li Ling ${ }^{2}$ \\ ${ }^{1}$ Laboratoire d'Enzymologie Moléculaire, Institut de Biologie Structurale Jean-Pierre Ebel, 41 rue Jules Horowitz, \\ 38027 Grenoble Cedex 1, France \\ ${ }^{2}$ Laboratoire de Microscopie Electronique Structurale, Institut de Biologie Structurale Jean-Pierre Ebel, 41 rue Jules Horowitz, \\ 38027 Grenoble Cedex 1, France \\ Correspondence should be addressed to Gérard J. Arlaud, arlaud.gerard@orange.fr
}

Received 21 October 2010; Accepted 18 December 2010

Academic Editor: Robert Salomon

Copyright ( $\odot 2011$ Gérard J. Arlaud et al. This is an open access article distributed under the Creative Commons Attribution License, which permits unrestricted use, distribution, and reproduction in any medium, provided the original work is properly cited.

\begin{abstract}
Several studies suggest that the complement system is involved in atherogenesis. To further investigate this question, we have studied the ability of native and modified forms of LDL to bind and activate $\mathrm{C} 1$, the complex protease that triggers the classical pathway of complement. Unlike native LDL, oxidized (oxLDL) and enzymatically modified (E-LDL) derivatives were both recognized by the C1q subunit of $\mathrm{C} 1$, but only E-LDL particles, obtained by sequential treatment with a protease and then with cholesterol esterase, had the ability to trigger $\mathrm{C} 1$ activation. Further investigations revealed that $\mathrm{C} 1 \mathrm{q}$ recognizes a lipid component of E-LDL. Several approaches, including reconstitution of model lipid vesicles, cosedimentation, and electron microscopy analyses, provided evidence that $\mathrm{C} 1$ binding to E-LDL particles is mediated by the C1q globular domain, which senses unesterified fatty acids generated by cholesterol esterase. The potential implications of these findings in atherogenesis are discussed.
\end{abstract}

\section{Introduction}

It is generally accepted that accumulation of low-density lipoproteins (LDLs) in the extracellular matrix of the blood vessels is the starting point of atherogenesis [1]. Nevertheless, how this leads to chronic inflammation and injury of the arterial wall is still poorly understood. The arterial intima is known to contain a number of oxidative agents and proteolytic enzymes, and these are thought to convert LDL particles into the lipid droplets and vesicles found during the early steps of atherogenesis [2-4]. Cell-induced oxidative modification or incubation in vitro with high concentrations of transition metals such as copper yields LDL derivatives endowed with atherogenic properties $[5,6]$. On the other hand, lipid particles enriched in unesterified cholesterol have been isolated from the arterial intima and found to be structurally similar to the LDL derivative (E-LDL) generated in vitro by sequential treatment with trypsin and cholesterol esterase (CEase) [7, 8]. It has been hypothesized that the oxidative and enzymatic modifications of LDL possibly complement each other, E-LDL being involved in the early steps of atherogenesis and oxidation playing a role in the progression of the disease [9].

Complement, known to be a major arm of innate immunity against pathogens, is also emerging as a potentially important factor of atherosclerosis [10]. That LDL derivatives have the ability to activate the complement system was initially described by Seifert and coworkers, who showed that cholesterol-containing lipid particles isolated from human atherosclerotic lesions activate complement to completion [11]. It was later reported by the same group that, unlike native LDL and oxidized LDL (oxLDL), E-LDL has the ability to activate complement both directly and in a C-reactive protein- (CRP-) dependent manner [12]. Triggering of the classical pathway of complement results from binding of the $\mathrm{C} 1$ complex, through its recognition subunit $\mathrm{Clq}$, to a variety of immune and nonimmune targets and elicits activation of its partner proteases C1r and C1s [13]. We have compared the ability of oxLDL and E-LDL derivatives to interact with the $\mathrm{C} 1$ complex, providing evidence that E-LDL efficiently 
activates $\mathrm{C} 1$ under conditions close to the physiological situation and is recognized by $\mathrm{C1q}$ [14]. More recently, we have shown that $\mathrm{Clq}$ recognizes E-LDL particles through unesterified fatty acids generated by cholesterol esterase treatment [15]. The aim of this paper is to briefly review these findings, which shed new light on the interactions between E-LDL and the classical pathway of complement and suggest a possible implication of these interactions in atherogenesis.

\section{Modified Lipoproteins Differentially Bind and Activate the C1 Complex of Complement}

The ability of native and modified forms of LDL to trigger activation of the $\mathrm{C} 1$ complex was tested in vitro by means of a $\mathrm{C} 1$ activation assay in the presence of excess $\mathrm{C} 1$ inhibitor in order to prevent spontaneous C1 activation [16]. Under these conditions, which are close to the physiological situation, purified native LDL did not significantly activate C1. Likewise, oxLDL had no significant activating effect at concentrations up to $1 \mu \mathrm{M}$, even though slight activation (about 10\%) was observed in the presence of CRP. In contrast, sequential treatment of native LDL with trypsin and then with CEase endowed the resulting E-LDL derivative with the ability to trigger efficient $\mathrm{C} 1$ activation [14]. The activation level at $1 \mu \mathrm{M}$ E-LDL reached about $60 \%$, and activation was not sensitive to CRP. Sequential treatment of LDL with trypsin and then CEase was critical to generate a C1-activating particle, and proteases such as plasmin and proteinase $K$ were as efficient as trypsin for this purpose.

The ability of $\mathrm{C} 1 \mathrm{q}$, the recognition unit of $\mathrm{C} 1$, to bind native LDL and its modified forms was investigated by surface plasmon resonance spectroscopy, using the lipoproteins as immobilized ligands and $\mathrm{Clq}$ as the soluble analyte. Clq did not show significant binding to native LDL but readily bound to both oxLDL and E-LDL. By recording binding curves at varying $\mathrm{Clq}$ concentrations, as illustrated in Figure 1 for E-LDL, the dissociation constants $\left(K_{D}\right)$ were determined. E-LDL and oxLDL each yielded values in the nanomolar range $(23-75 \mathrm{nM})$, indicating that $\mathrm{C} 1 \mathrm{q}$ binds both derivatives with high affinity [14].

In subsequent experiments [15], a series of proteolytic enzymes known to be present in atherosclerotic lesions were tested for their ability to generate C1-activating ELDL particles when used in conjunction with CEase. As shown by SDS-PAGE analysis, in addition to trypsin, plasmin, kallikrein, chymase, and thrombin all extensively split the apolipoprotein B-100 (Apo-B100) of LDL, whereas tryptase, and matrix metalloprotease- 2 had milder effects. Subsequent incubation with CEase revealed that samples initially treated with plasmin, thrombin, tryptase, and matrix metalloprotease-2 displayed a C1-activating ability comparable to that obtained using trypsin, whereas chymase and kallikrein had moderate or no effect. Thus, initial treatment of LDL with a protease was clearly a prerequisite to generate $\mathrm{C} 1$-activating particles, but there was no strict correlation between the extent of Apo-B100 degradation and the resulting $\mathrm{C} 1$ activation extent. Increasing the CEase concentration and the incubation time both readily increased the $\mathrm{Cl}$ activating ability of the resulting LDL particles, yielding

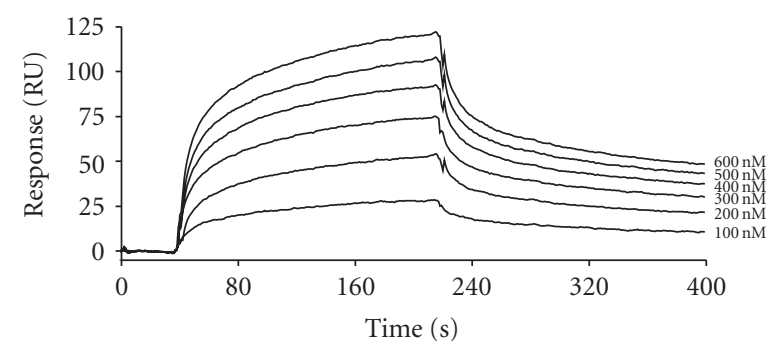

FIGURE 1: Analysis by surface plasmon resonance spectroscopy of the interaction between $\mathrm{Clq}$ and immobilized E-LDL. The E-LDL derivative (14,000 resonance units) was immobilized chemically on the surface of a CM5 sensor chip (GE Healthcare) and allowed to bind to increasing concentrations of soluble C1q (100-600 nM). The $K_{D}$ value was determined from the ratio of the dissociation and association rate constants $\left(k_{\text {off }} / k_{\text {on }}\right)$ (taken from [14], with permission).

complete $\mathrm{C} 1$ activation after incubation of trypsin-treated LDL with 320 milliunits/mL CEase for $18 \mathrm{~h}$ at $37^{\circ} \mathrm{C}$ [15]. Thus, subsequent modification of trypsin-treated LDL with CEase was clearly a determinant step for generating particles recognized by $\mathrm{C} 1 \mathrm{q}$ and exhibiting $\mathrm{C} 1$-activating ability. In contrast, treatment of native LDL with phospholipase $\mathrm{A}_{2}$ or sphingomyelinase did not yield significant $\mathrm{C} 1$ activation.

\section{C1q Recognizes a Lipid Component of E-LDL}

As a first step towards identification of the LDL component(s) recognized by C1q, the lipid fractions of native LDL, trypsin-treated LDL, and E-LDL were extracted and used to prepare vesicles which were then tested for their ability to activate C1 [15]. As shown in Figure 2, vesicles containing the lipid fraction of unmodified or trypsin-treated LDL did not yield $\mathrm{C} 1$ activation. In contrast, vesicles prepared from LDL samples treated with trypsin and then incubated with CEase for increasing periods at $37^{\circ} \mathrm{C}$ developed increasing $\mathrm{C} 1$ activating ability, to reach about $90 \%$ activation after incubation with 320 milliunits CEase for $16 \mathrm{~h}$. In contrast, the protein component of this sample left after lipid extraction did not induce $\mathrm{C} 1$ activation. Likewise, native or trypsintreated ApoB-100 had no activating effect.

As measured by reverse-phase HPLC, the lipid fraction of native LDL contained a small amount of unesterified cholesterol $(398 \pm 50 \mathrm{nmol} / \mathrm{mg}$ of protein), as well as the cholesteryl esters characteristic of LDL $(22: 6,20: 4,18: 2$, $18: 1$, and $16: 0)$. Incubation overnight at $37^{\circ} \mathrm{C}$ led to nearly complete disappearance of the cholesteryl ester peaks, with concomitantly a raise of unesterified cholesterol to $3140 \pm$ $160 \mathrm{nmol} / \mathrm{mg}$ of protein. Analysis of the lipid fraction of the E-LDL particles generated upon incubation for increasing periods with CEase showed a good correlation between the content in unesterified cholesterol and the extent of $\mathrm{C} 1$ activation. This suggested that cholesterol itself, or fatty acids, or both of these molecules resulting from CEase treatment could be recognized by $\mathrm{C} 1 \mathrm{q}$. 


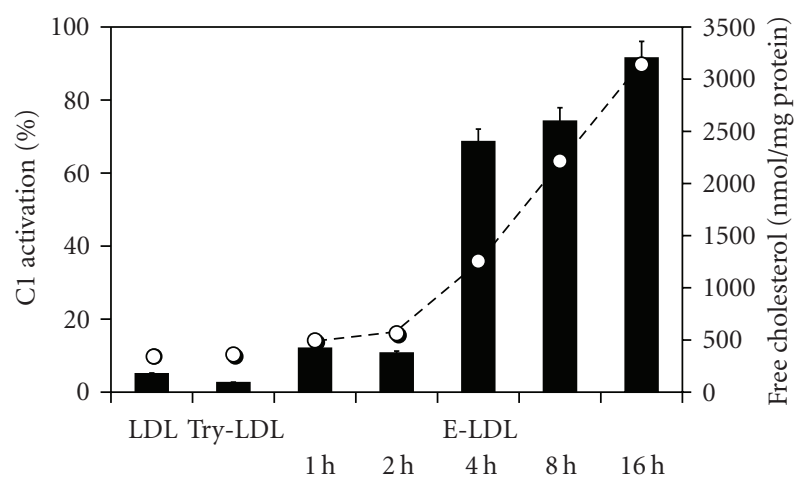

FIgURE 2: C1 activation by E-LDL particle: correlation with the amount of unesterified cholesterol generated. LDL $(1 \mathrm{mg} / \mathrm{mL})$ was treated with $20 \mu \mathrm{g} / \mathrm{mL}$ trypsin for $2 \mathrm{~h}$ at $37^{\circ} \mathrm{C}$ and then with 320 milliunits $/ \mathrm{mL}$ CEase for the indicated periods at $37^{\circ} \mathrm{C}$. The lipid fraction from each sample was extracted and incorporated into vesicles. Each vesicle was tested for its C1-activating ability (black bars). The cholesterol content of each lipid fraction (open circles) was determined by reverse-phase HPLC. LDL: native LDL; Try-LDL: trypsin-treated LDL (taken from [15], with permission).

\section{C1q Recognizes E-LDL through Unesterified Fatty Acids Generated by CEase}

To identify the lipid component(s) of E-LDL recognized by $\mathrm{Clq}$, E-LDL was treated with methyl- $\beta$-cyclodextrin $(\mathrm{MBCD})$, a reagent known to extract unesterified cholesterol from membranes [15]. Incubation of E-LDL particles with increasing concentrations of $\mathrm{MBCD}$ for $1 \mathrm{~h}$ at $37^{\circ} \mathrm{C}$ progressively abolished their $\mathrm{C} 1$-activating ability. However, parallel analyses by thin-layer chromatography clearly indicated that MBCD not only depleted free cholesterol but also removed unesterified fatty acids, yielding in both cases $>70 \%$ depletion at an MBCD concentration of $2.25 \mathrm{mM}$.

Incubation of the E-LDL particles with increasing concentrations of human serum albumin, a reagent known to remove unesterified fatty acids, decreased their C1-activating ability in a dose-dependent fashion, resulting in $>60 \%$ inhibition upon treatment with $10 \%$ (w/v) albumin. Analysis by thin-layer chromatography revealed that the $\mathrm{C} 1$-activating ability of the samples roughly correlated with the extent of unesterified fatty acid removal, whereas free cholesterol was not depleted under these conditions. These data provided a first indication that binding of the $\mathrm{C} 1 \mathrm{q}$ subunit of $\mathrm{C} 1$ to ELDL particles involved the unesterified fatty acids generated by CEase treatment.

To further investigate this question, model vesicles containing phosphatidylcholine (PC) and increasing proportions of unesterified cholesterol and/or linoleic acid were prepared and tested for their ability to activate C1 [15]. Increasing the amount of linoleic acid increased the $\mathrm{C} 1$ activating potential of the vesicles, yielding about $65 \%$ activation at a $1: 1$ linoleic acid: $\mathrm{PC}$ ratio, and then had the opposite effect at a 2:1 ratio. In contrast, increasing both linoleic acid and cholesterol dose-dependently increased the ability of the vesicles to activate $\mathrm{C} 1$, yielding full activation at a $2: 2: 1$ linoleic acid: cholesterol: PC ratio. Further analyses

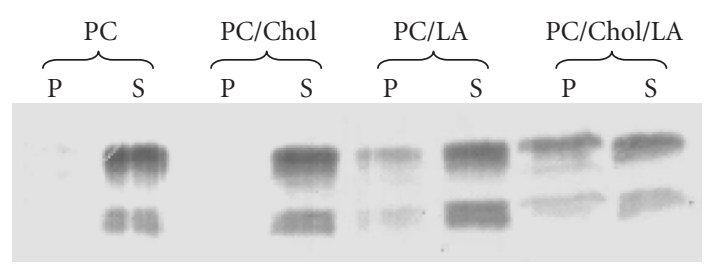

Figure 3: Cosedimentation analysis of the interaction between the $\mathrm{Clq}$ globular domain and artificial lipid vesicles. Vesicles were prepared from PC alone, PC: cholesterol $(1: 2, \mathrm{w} / \mathrm{w}), \mathrm{PC}$ : linoleic acid $(1: 2, \mathrm{w} / \mathrm{w})$, or PC: cholesterol: linoleic acid $(1: 2: 2, \mathrm{w} / \mathrm{w} / \mathrm{w})$. Each type of vesicle was incubated with the $\mathrm{Clq}$ globular domain (C1q GR), and binding was measured from the relative amount of C1q GR associated with the vesicles in the ultracentrifugation pellet, as determined by SDS-PAGE analysis of the pellet $(\mathrm{P})$ and supernatant (S) fractions. Chol: cholesterol; LA: linoleic acid; PC: phosphatidylcholine (taken from [15], with permission).

were conducted using lipid vesicles containing PC plus either cholesterol, or linoleic acid, or both cholesterol and linoleic acid. After incubation of these vesicles with the C1q globular domain (C1q GR), interaction was assessed by cosedimentation analysis from the relative amount of $\mathrm{Clq}$ GR sedimenting with the vesicles in the ultracentrifugation pellet (Figure 3). No binding to vesicles containing PC alone or PC + cholesterol was detected. A slight but significant binding (about 20\%) was observed using vesicles containing $\mathrm{PC}+$ linoleic acid, and this value increased to $46 \%$ when both cholesterol and linoleic acid were present (Figure 3). Analysis of the pellets revealed that linoleic acid was more efficiently incorporated into the vesicles in the presence of cholesterol. It became clear, therefore, that linoleic acid was the only ligand recognized by $\mathrm{Clq}$, the enhancing effect exerted by cholesterol being due to its ability to facilitate incorporation of free fatty acids into the vesicles. Another indication from the cosedimentation experiments was that recognition by Clq of the fatty acids of E-LDL was mediated by its globular (GR) domains.

\section{Electron Microscopy of E-LDL-Bound C1q Molecules}

Negative staining electron microscopy was used to visualize interaction of the whole C1q molecule with E-LDL particles. Bound $\mathrm{Clq}$ molecules were clearly seen to interact with the particles through their globular domains. In some cases, most of the 6 globular domains were found to follow the curvature of the particles (Figure 4(a)), whereas other C1q molecules interacted through only a few heads (Figure 4(b)). Some free $\mathrm{Clq}$ molecules not interacting with E-LDL particles could also be identified (Figure 4(c)).

\section{Discussion}

The various experiments carried out in the past years provide clear experimental evidence that, in contrast to native LDL and oxLDL, E-LDL is endowed with the ability to activate $\mathrm{C} 1$, the multimolecular protease that triggers the classical 

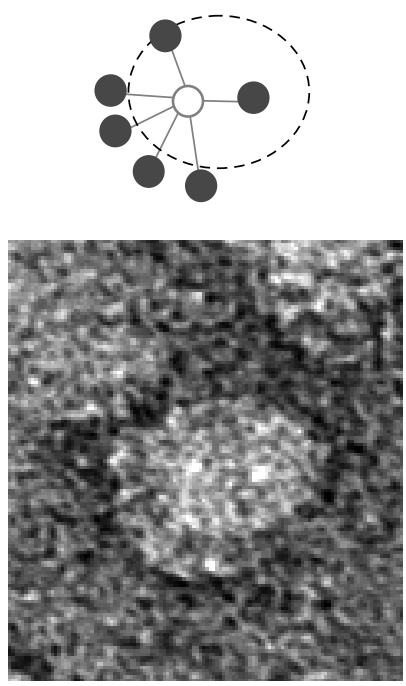

(a)
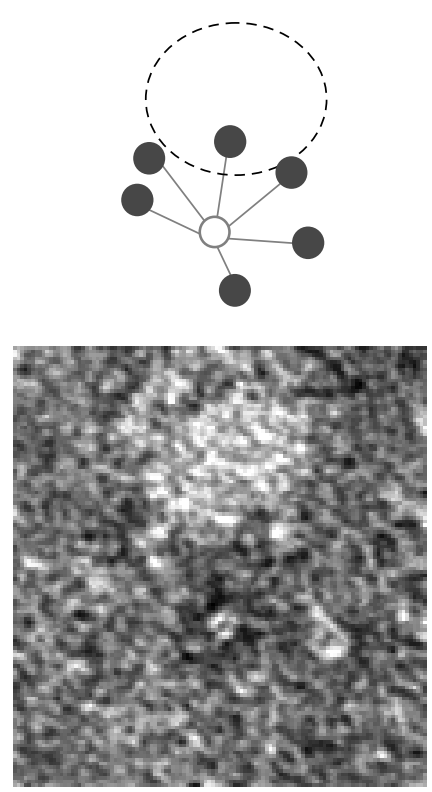

(b)
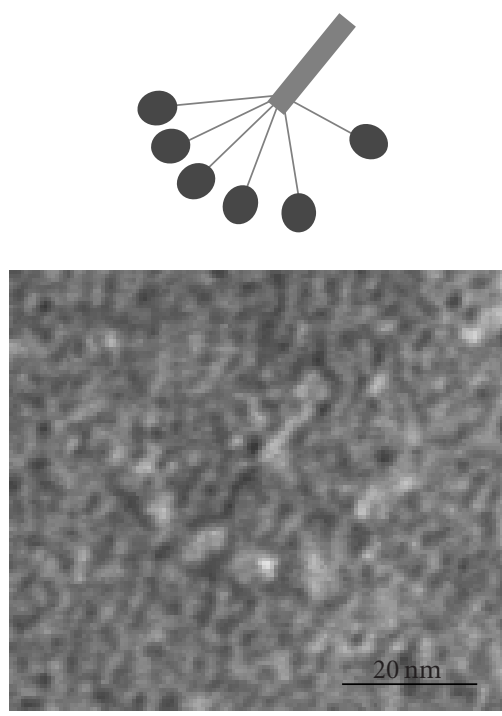

(c)

Figure 4: Electron micrographs of E-LDL-bound C1q molecules. (a, b) Examples of C1q molecules interacting through most of their globular domains (a) or only a few (b). (c) Representative example of a free C1q molecule (modified from [15], with permission).

pathway of complement. The observation that native LDL particles do not activate $\mathrm{C} 1$ is consistent with the fact that these are not recognized by C1q. In contrast, oxLDL particles are sensed by C1q, with a binding affinity similar to that determined for E-LDL; however, they do not trigger C1 activation. It appears likely therefore that oxidation generates $\mathrm{C} 1 \mathrm{q}$ binding sites on the LDL surface, but these are distributed in a manner that does not allow $\mathrm{C} 1$ activation. An alternative explanation is that $\mathrm{C} 1$ activation by oxLDL is efficiently prevented by $\mathrm{C} 1$ inhibitor, as observed for other "weak" nonimmune activators of $\mathrm{C} 1$ such as DNA and heparin $[17,18]$. In contrast, the fact that E-LDL behaves as a potent $\mathrm{Cl}$ activator in the presence of excess $\mathrm{C} 1$ inhibitor strongly suggests that this process observed in vitro is biologically relevant.

A further major conclusion from these investigations is that $\mathrm{C} 1$ binding to $\mathrm{E}-\mathrm{LDL}$ particles is mediated by the $\mathrm{C} 1 \mathrm{q}$ globular domains and that these recognize the unesterified fatty acid molecules generated by CEase treatment. The conversion of native LDL into a particle endowed with C1-activating ability clearly requires prior treatment with a proteolytic enzyme and then with CEase. In addition to trypsin used as a model, several proteases (plasmin, matrix metalloproteases-2, thrombin, tryptase) present in the atherosclerotic lesions $[19,20]$ are able to generate ELDL particles with high $\mathrm{C} 1$-activating ability. Considering that CEase itself is present in the arterial intima $[8,21]$, it appears likely that C1-activating E-LDL particles are indeed generated in vivo. In agreement with this hypothesis, electron microscopy analyses show that extensive hydrolysis of cholesteryl esters by CEase generates large liposome-like particles [15] similar to those previously described by Chao et al. [8] and reminiscent of the lipid particles observed in atherosclerotic lesions [7, 22].

Proteolysis of ApoB-100 appears necessary to allow access of cholesteryl ester molecules to CEase. Although no strict correlation was observed between the extent of ApoB-100 degradation and the C1-activating ability of the resulting particle, it should be emphasized that the SDSPAGE technique used only gives a rough account of the extent of LDL degradation. The most likely hypothesis is therefore that proteolytic treatment of the LDL particle disorganizes ApoB-100 [8], hence allowing CEase to gain access to the underlying hydrophobic core containing cholesteryl esters and triglycerides, resulting in the production of large amounts of cholesterol and unesterified fatty acids.

How C1q senses fatty acids on the E-LDL surface remains to be determined at the molecular level. However, C1q binding is clearly mediated by its globular domain, indicating that, in addition to its numerous ligands already identified [23], this domain recognizes the polar head of fatty acids and therefore possesses a binding site for carboxyl groups. This is consistent with the known ability of $\mathrm{Clq}$ to bind polyanionic ligands [24], considering that treatment with CEase converts LDL particles into polyanions. These investigations provide clear evidence that E-LDL is recognized by $\mathrm{Clq}$, further establishing the role of this protein as a sensor of altered self-components. Another modified form of LDL, acetylated $\mathrm{LDL}$, was recently shown to be recognized by $\mathrm{Clq}$, as well as by two other innate immune recognition proteins involved in activation of the lectin, pathway of complement, mannanbinding lectin, and L-ficolin $[25,26]$.

The observation that E-LDL triggers activation of the classical complement pathway provides further support for 
an implication of complement in atherogenesis. It now remains to be established whether complement activation has a protective effect through the removal of E-LDL particles from atherosclerotic plaques or is proatherogenic because of the intrinsic ability of the terminal complement pathway to generate inflammatory mediators.

\section{References}

[1] A. J. Lusis, "Atherosclerosis," Nature, vol. 407, no. 6801, pp. 233-241, 2000.

[2] D. Steinberg, "Low density lipoprotein oxidation and its pathobiological significance," Journal of Biological Chemistry, vol. 272, no. 34, pp. 20963-20966, 1997.

[3] S. Bhakdi, B. Dorweiler, R. Kirchmann et al., "On the pathogenesis of atherosclerosis: enzymatic transformation of human low density lipoprotein to an atherogenic moiety," Journal of Experimental Medicine, vol. 182, no. 6, pp. 19591971, 1995.

[4] M. Torzewski, P. Suriyaphol, K. Paprotka et al., "Enzymatic modification of low-density lipoprotein in the arterial wall: a new role for plasmin and matrix metalloproteinases in atherogenesis," Arteriosclerosis, Thrombosis, and Vascular Biology, vol. 24, no. 11, pp. 2130-2136, 2004.

[5] D. Steinberg, S. Parthasarathy, T. E. Carew, J. C. Khoo, and J. L. Witztum, "Beyond cholesterol: modifications of low-density lipoprotein that increase its atherogenicity," New England Journal of Medicine, vol. 320, no. 14, pp. 915-924, 1989.

[6] E. Wieland, S. Parthasarathy, and D. Steinberg, "Peroxidasedependent metal-independent oxidation of low density lipoprotein in vitro: a model for in vivo oxidation?" Proceedings of the National Academy of Sciences of the United States of America, vol. 90, no. 13, pp. 5929-5933, 1993.

[7] F. F. Chao, L. M. Amende, E. J. Blanchette-Mackie et al., "Unesterified cholesterol-rich lipid particles in atherosclerotic lesions of human and rabbit aortas," American Journal of Pathology, vol. 131, no. 1, pp. 73-83, 1988.

[8] F. F. Chao, E. J. Blanchette-Mackie, V. V. Tertov, S. I. Skarlatos, Y. J. Chen, and H. S. Kruth, "Hydrolysis of cholesteryl ester in low density lipoprotein converts this lipoprotein to a liposome," Journal of Biological Chemistry, vol. 267, no. 7, pp. 4992-4998, 1992.

[9] M. Torzewski and K. J. Lackner, "Initiation and progression of atherosclerosis-enzymatic or oxidative modification of low-density lipoprotein?" Clinical Chemistry and Laboratory Medicine, vol. 44, no. 12, pp. 1389-1394, 2006.

[10] D. O. Haskard, J. J. Boyle, and J. C. Mason, "The role of complement in atherosclerosis," Current Opinion in Lipidology, vol. 19, no. 5, pp. 478-482, 2008.

[11] P. S. Seifert, F. Hugo, J. Tranum-Jensen, U. Zahringer, M. Muhly, and S. Bhakdi, "Isolation and characterization of a complement-activating lipid extracted from human atherosclerotic lesions," Journal of Experimental Medicine, vol. 172, no. 2, pp. 547-557, 1990.

[12] S. Bhakdi, M. Torzewski, K. Paprotka et al., "Possible protective role for C-reactive protein in atherogenesis: complement activation by modified lipoproteins halts before detrimental terminal sequence," Circulation, vol. 109, no. 15, pp. 18701876, 2004.

[13] C. Gaboriaud, N. M. Thielens, L. A. Gregory, V. Rossi, J. C. Fontecilla-Camps, and G. J. Arlaud, "Structure and activation of the C1 complex of complement: unraveling the puzzle," Trends in Immunology, vol. 25, no. 7, pp. 368-373, 2004.
[14] A. Biró, N. M. Thielens, L. Cervenák, Z. Prohászka, G. Füst, and G. J. Arlaud, "Modified low density lipoproteins differentially bind and activate the C1 complex of complement," Molecular Immunology, vol. 44, no. 6, pp. 1169-1177, 2007.

[15] A. Biro, W. L. Ling, and G. J. Arlaud, "Complement protein clq recognizes enzymatically modified low-density lipoprotein through unesterified fatty acids generated by cholesterol esterase," Biochemistry, vol. 49, no. 10, pp. 2167-2176, 2010.

[16] P. Tacnet-Delorme, S. Chevallier, and G. J. Arlaud, " $\beta$ amyloid fibrils activate the $\mathrm{C} 1$ complex of complement under physiological conditions: evidence for a binding site for $\mathrm{A} \beta$ on the C1q globular regions," Journal of Immunology, vol. 167, no. 11, pp. 6374-6381, 2001.

[17] R. J. Ziccardi, "A new role for C1-inhibitor in homeostasis: control of activation of the first component of human complement," Journal of Immunology, vol. 128, no. 6, pp. 2505-2508, 1982.

[18] V. Garlatti, A. Chouquet, T. Lunardi et al., "Cutting edge: C1q binds deoxyribose and heparan sulfate through neighboring sites of its recognition domain," Journal of Immunology, vol. 185, no. 2, pp. 808-812, 2010.

[19] M. O. Pentikäinen, E. M. P. Lehtonen, and P. T. Kovanen, "Aggregation and fusion of modified low density lipoprotein," Journal of Lipid Research, vol. 37, no. 12, pp. 2638-2649, 1996.

[20] K. Oorni, M. O. Pentikainen, M. Ala-Korpela, and P. T. Kovanen, "Aggregation, fusion, and vesicle formation of modified low density lipoprotein particles: molecular mechanisms and effects on matrix interactions," Journal of Lipid Research, vol. 41, no. 11, pp. 1703-1714, 2000.

[21] H. V. Kothari, M. J. Bonner, and B. F. Miller, "Cholesterol ester hydrolase in homogenates and lysosomal fractions of human aorta," Biochimica et Biophysica Acta, vol. 202, no. 2, pp. 325$331,1970$.

[22] N. Simionescu, E. Vasile, F. Lupu et al., "Prelesional events in atherogenesis: accumulation of extracellular cholesterolrich liposomes in the arterial intima and cardiac valves of the hyperlipidemic rabbit," American Journal of Pathology, vol. 123, no. 1, pp. 109-125, 1986.

[23] U. Kishore, C. Gaboriaud, P. Waters et al., "C1q and tumor necrosis factor superfamily: modularity and versatility," Trends in Immunology, vol. 25, no. 10, pp. 551-561, 2004.

[24] N. R. Cooper, "The classical complement pathway: activation and regulation of the first complement component," Advances in Immunology, vol. 37, pp. 151-216, 1985.

[25] D. A. Fraser and A. J. Tenner, "Innate immune proteins C1q and mannan-binding lectin enhance clearance of atherogenic lipoproteins by human monocytes and macrophages," Journal of Immunology, vol. 185, no. 7, pp. 3932-3939, 2010.

[26] J. Faro, Y. Chen, P. Jhaveri et al., "L-Ficolin binding and lectin pathway activation by acetylated low-density lipoprotein," Clinical and Experimental Immunology, vol. 151, no. 2, pp. 275-283, 2008. 

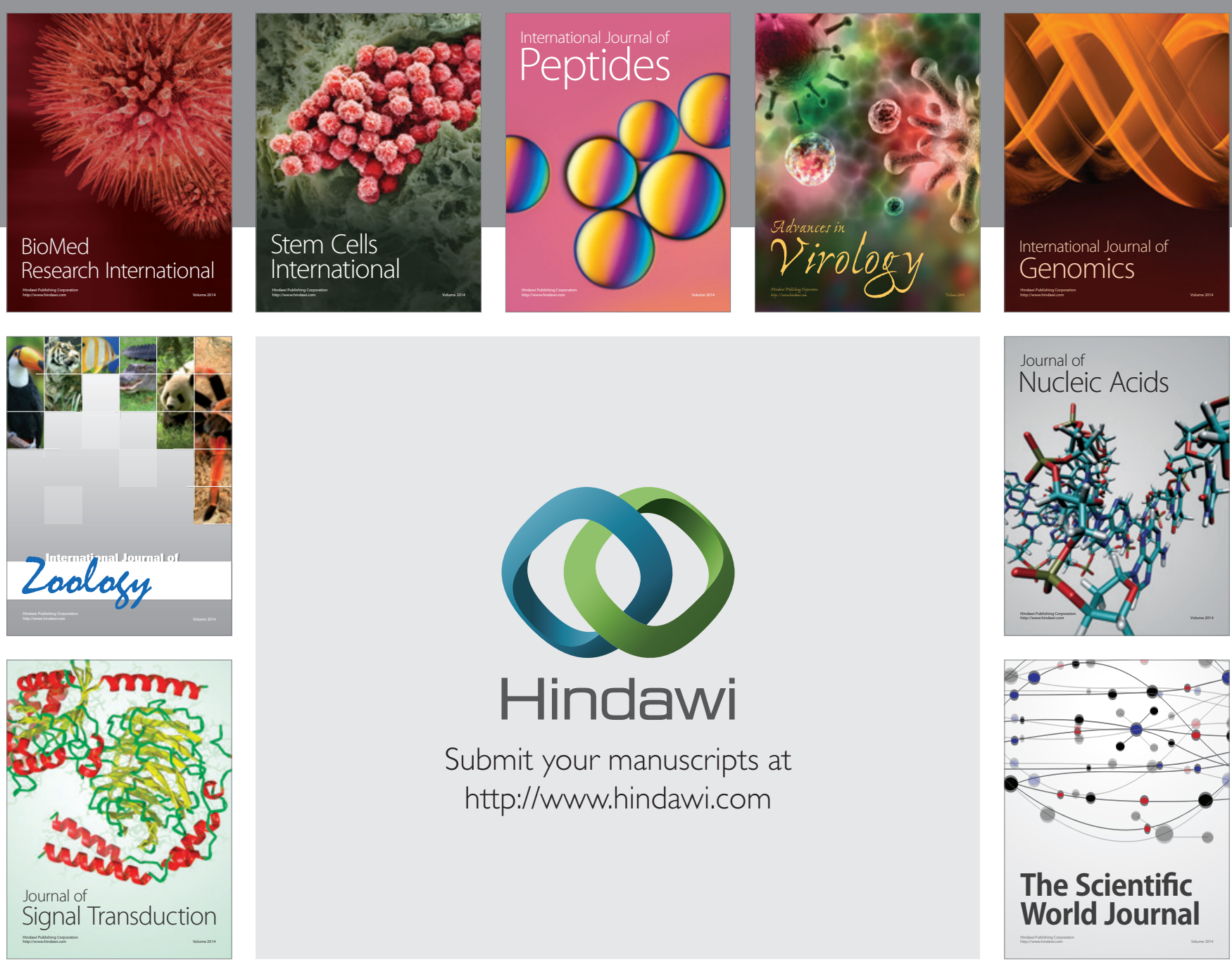

Submit your manuscripts at

http://www.hindawi.com
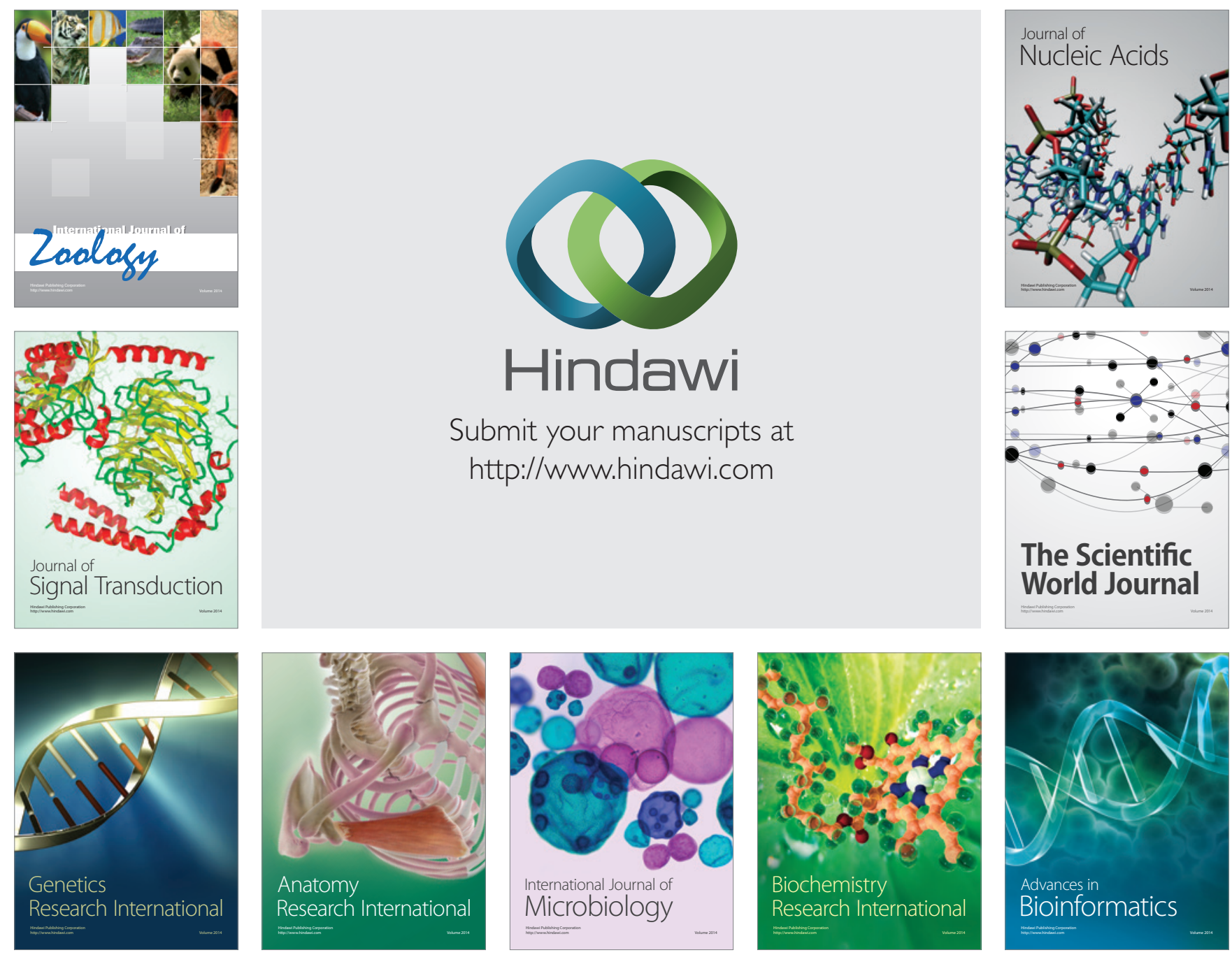

The Scientific World Journal
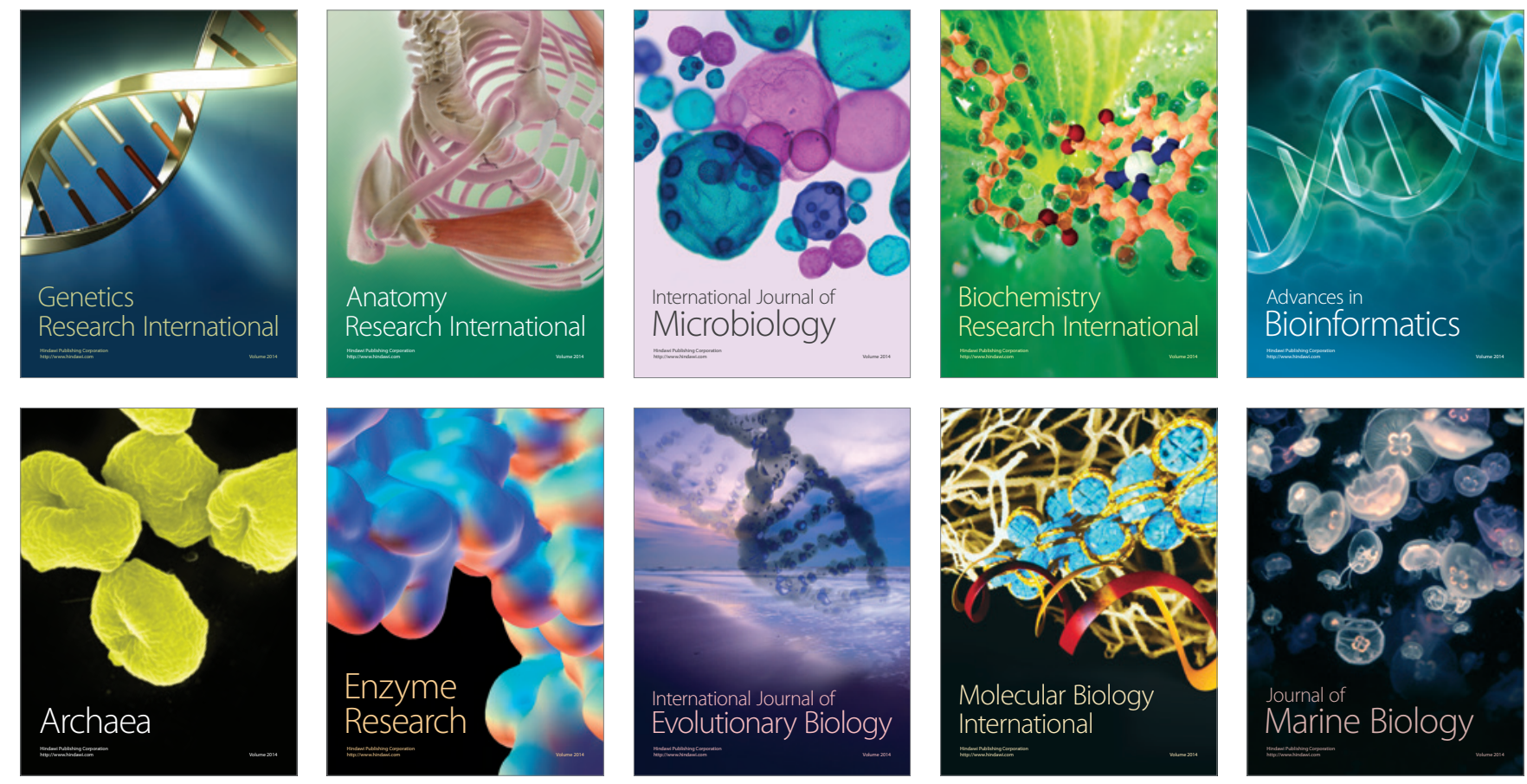